(C) 2008 The Japan Society of Applied Physics

\title{
Correlation Field Analysis of Magnetoresistance of GaN/AIGaN Heterostructure Grown on Si Substrate
}

\author{
Li-Hung LiN*, Shiou-Shian Han, Kui-Ming Chen, Zhi-Yao Zhang ${ }^{1}$, Kuang Yao Chen ${ }^{1}$, J. Z. HuAng ${ }^{1}$, \\ Zhi-Hao Sun ${ }^{1}$, C.-T. LIANG ${ }^{1,2}$, N. C. CHEN ${ }^{3}$, P. H. CHANG ${ }^{3}$, and Chin-An CHANG ${ }^{3}$
}

\author{
Institute of Optoelectronics and Solid State Electronics, National Chiayi University, Chiayi 60004, Taiwan \\ ${ }^{1}$ Department of Physics, National Taiwan University, Taipei 10617, Taiwan \\ ${ }^{2}$ Institute of Astrophysics, National Taiwan University, Taipei 10617, Taiwan \\ ${ }^{3}$ Institute of Electro-Optical Engineering, Chang Gang University, Taoyuan 300, Taiwan
}

(Received July 12, 2007; revised November 30, 2007; accepted February 4, 2008; published online June 13, 2008)

\begin{abstract}
We analyze the conductance fluctuations for a bulk two-dimensional electron system in a $\mathrm{GaN} / \mathrm{Al}_{0.15} \mathrm{Ga}_{0.85} \mathrm{~N}$ heterostructure grown on a p-type $\mathrm{Si}(111)$ substrate, and calculate the correlation field $B_{\mathrm{c}}$ with the autocorrelation function $F(\Delta B)=$ $\langle\delta G(B+\Delta B)-\delta G(B)\rangle$, in the temperature range $0.27 \leq T \leq 3 \mathrm{~K}$, which is employed as a self-thermometer to obtain the effective carrier temperature $T_{\mathrm{e}}$ as a function of the measurement current $I$. We find that the temperature dependence agrees well with simple expectations for universal conductance fluctuations in metals, while the observed amplitude is reduced. In the study of hot-carrier phenomena, we also obtain $T_{\mathrm{e}} \sim I^{0.5}$, which agrees with some theoretical prediction.
\end{abstract}

[DOI: $10.1143 /$ JJAP.47.4623]

KEYWORDS: GaN, HEMT, conductance fluctuation, hot carrier, current scaling

\section{Introduction}

$\mathrm{GaN} / \mathrm{AlGaN}$ heterostructures have been attracting a great deal of experimental and theoretical interest because of their applications in light-emitting diodes, ${ }^{1,2)}$ high-power microwave devices, ${ }^{3)}$ high-frequency field-effect transistors, ${ }^{4)}$ semiconductor lasers, ${ }^{5,6)}$ and high-electron-mobility transistors. $^{7-10)} \mathrm{GaN} / \mathrm{AlGaN}$ heterostructures are usually grown on sapphire. However, Si can also be regarded as an alternative to commercial sapphire substrates for GaN-based semiconductor devices because of its low cast and large wafer size. Because of the large lattice (17\%) and thermal $(54 \%)$ mismatches between $\mathrm{Si}$ and $\mathrm{GaN}$, high-quality $\mathrm{GaN}$ films are extremely difficult to grow on $\mathrm{Si}$. An intermediate nucleation layer of AlN grown on top of Si can greatly improve the quality of subsequently grown GaN films. ${ }^{112)}$ Recently, it has been shown that by introducing an ultrathin $\mathrm{SiN}_{x}$ film during crystal growth, the Hall mobility of a $\mathrm{GaN} / \mathrm{Al}_{0.15^{-}}$ $\mathrm{Ga}_{0.85} \mathrm{~N}$ heterostructure on $\mathrm{Si}$ could be markedly increased (>3 times). ${ }^{13-15)}$ These $\mathrm{GaN} / \mathrm{Al}_{0.15} \mathrm{Ga}_{0.85} \mathrm{~N}$ heterostructures grown on $\mathrm{Si}$ are compatible with complementary metaloxide-semiconductor technology and have promising device applications, making it useful in understanding the fundamental physics of these heterostructures.

Electron heating is a central problem in semiconductor devices, both in device applications and in research on solid state physics. Hot carriers are created when carriers are accelerated by an electric field if the energy transport between carriers and metal lattice is weaker than that from the lattice to the surroundings. At liquid-helium temperature, electrically accelerated carriers can be heated to a higher temperature, $T_{\mathrm{e}}$, while phonons stay at a cooler temperature, $T_{\mathrm{L}}$, in a ballistic or quasi-ballistic transport system, which is caused by the carrier-phonon energy exchange rate being lower than that at higher temperature. In some theoretical and experimental studies of GaAs/AlGaAs heterostructures and other two-dimensional electron systems (2DESs), the power law $T_{\mathrm{e}} \sim I^{\alpha}$ arises in hot-carrier phenomena, where $I$

*E-mail address: lihung@mail.ncyu.edu.tw is the current. ${ }^{16,17)}$ The widths of peaks or derivatives of plateaus in magnetoresistance (MR) are frequently used as self-thermometers for estimating carrier temperature $T_{\mathrm{e}}$.

Conductance fluctuations may be one of the most prominent phenomena in mesoscopic structures. For diffusive conducting systems at sufficiently low temperatures, these so-called universal conductance fluctuations (UCFs) have a characteristic amplitude, $\sim e^{2} / h$, independent of sample size and material, and they are generally thought to be well understood. ${ }^{18)}$ Similar conductance fluctuations are also observed and discussed in mesoscopic quantum Hall $(\mathrm{QH})$ systems, ${ }^{19-28)}$ quantum wires, ${ }^{29-32)}$ quantum point contacts, ${ }^{33,34)}$ open cavity (quantum dot) systems, ${ }^{35-42)}$ and loop systems. ${ }^{43)}$ Interpretations of different experiments include the modification of UCF in high field, ${ }^{19,20)}$ the tunneling between opposite edge states through bulk inhomogeneities, ${ }^{22-24)}$ the influences of charging effects, ${ }^{25,26)}$ and the network of compressible-incompressible regions. ${ }^{27)}$ In these studies of conductance fluctuations, the autocorrelation function $F(\Delta B)=\langle\delta G(B+\Delta B)-\delta G(B)\rangle$ of $\mathrm{MR}$ fluctuations $\delta G(B)$ is frequently employed, where the angle brackets denote the average over all magnetic fields. Some physical amounts can be estimated by the analyses of conductance fluctuations, including the correlation field $B_{\mathrm{c}}$, which is defined as $F\left(B_{\mathrm{c}}\right)=F(0) / 2$. The dephasing length $L_{\varphi}(T)$ of carriers, as an example, can be estimated with $B_{\mathrm{c}}(T)=\phi_{0} /\left[L_{\varphi}(T)\right]^{2}$, where the magnetic flux quantum $\phi_{0}$ is given by $h / e^{21)}$

In this study, we use $B_{c}$ for studying the $\mathrm{GaN} / \mathrm{Al}_{0.15^{-}}$ $\mathrm{Ga}_{0.85} \mathrm{~N}$ heterostructure on $\mathrm{Si}$ and use $B_{\mathrm{c}}$ as a selfthermometer to find the scaling relation between the measurement current $I$ and the effective carrier temperature $T_{\mathrm{e}}$, and discuss the hot-carrier phenomena in the system. Many works have been conducted on microscopic scattering processes these years. A model of scaling in the integer quantum Hall region when a current $I$ is applied was introduced by Polyakov and Shklovskii, ${ }^{44)}$ and a recent study showed that an effective electron temperature $T_{\mathrm{e}} \sim I^{0.5}$ can be deduced by combining temperature scaling and current scaling of the amplitude or peak broadening of Shubnikov- 
de Haas ( $\mathrm{SdH}$ ) oscillation in MR. ${ }^{16,17)}$ The experimental results $T_{\mathrm{e}} \sim I^{0.5}$ have been reported for GaAs/AlGaAs and InGaAs/InP 2DESs. ${ }^{16,17,45)}$

\section{Experimental Methods}

The samples studied in this work were grown using metalorganic vapor phase epitaxy in an Aixtron RF-S system. ${ }^{14)}$ The following layer sequences was grown on p-type Si(111) substrates: 2.5-nm low-temperature (LT) AlN, 2.5nm high-temperature (HT) AlN, an ultra-thin $\mathrm{SiN}_{x}$ (only in sample B), 350-nm GaN, 12-nm LT AlN, 12-nm HT AlN, 400-nm GaN, and 30-nm $\mathrm{Al}_{0.15} \mathrm{Ga}_{0.85} \mathrm{~N}$, where LT and HT AlN layers correspond to AlN layers grown at 620 and $1050{ }^{\circ} \mathrm{C}$, respectively. In this work, all the GaN and AlGaN layers were grown at $1050{ }^{\circ} \mathrm{C}$ with a Ga polarity. The $\mathrm{SiN}_{x}$ treatment of sample B was introduce by $\mathrm{SiH}_{4}$ flow of 50 sccm diluted to $100 \mathrm{ppm}$ with $\mathrm{H}_{2}$. Growth pressure and temperature were kept at $100 \mathrm{mbar}$ and $1050^{\circ} \mathrm{C}$, respectively, for $5 \mathrm{~s}$. The $\mathrm{SiN}_{x}$ treatment is believed to help reduce the dislocation density in the $\mathrm{GaN}$ layer, improving both the quality of the GaN layer and the mobility of the 2DES in sample B. The carrier concentrations of the 2DESs in samples $A$ and $B$ are $9.2 \times 10^{12}$ and $9.4 \times 10^{12} \mathrm{~cm}^{-2}$ respectively, with Hall mobilities of $\sim 700$ and $\sim 2500$ $\mathrm{cm}^{2} \cdot \mathrm{V}^{-1} \cdot \mathrm{s}^{-1}$ respectively at $T=0.27 \mathrm{~K}$. The carrier concentrations of our systems appear to be slightly higher than that of an undoped $\mathrm{GaN} / \mathrm{AlGaN}$ heterostructure grown on sapphire, which may be caused by the stronger strain introduced during the crystal growth. The four-terminal MR of $4 \times 4 \mathrm{~mm}^{2}$ 2DES bulks in the van der Pauw method was measured in a ${ }^{3} \mathrm{He}$ cryostat over a wide range of temperatures $0.27 \leq T \leq 70 \mathrm{~K}$, by using standard phase-sensitive ac lock-in techniques, and the magnetic field $-15 \leq B \leq$ $15 \mathrm{~T}$ was generated by a superconducting magnet, which applies magnetic field perpendicular to the substrates.

\section{Results and Discussion}

The longitudinal MR of sample A (without $\mathrm{SiN}_{x}$ treatment) shows no clear signal of $\mathrm{SdH}$ oscillations even at low temperatures and high magnetic fields, which implies that the Landau level in this sample may be broaden by defects. On the other hand, sample B (with $\mathrm{SiN}_{x}$ treatment) shows $\mathrm{SdH}$ oscillations at low temperatures $<10 \mathrm{~K}$ (Fig. 1). The enhancement of the what of sample B has also been studied by transmission electron microscopy in our previous work, where sample B shows a lower dislocation density than sample A. We concentrate on the MR analysis of sample B in the $\mathrm{QH}$-conducting regime where $\mathrm{SdH}$ oscillations can be observed.

After the subtraction of the MR background of sample B, MR fluctuations $\delta G(B)$ were obtained in both the QHconducting regime $(B>4.5 \mathrm{~T})$ and the insulating regime $(B<4.5 \mathrm{~T})$. The MR fluctuations are $\sim(0.01) e^{2} / h$, smaller than typical UCF for one order (Fig. 1, inset). On the other hand, MR fluctuations usually average out in a homogeneous heterostructure, but temperature-dependent MR fluctuations are observed in sample B, which may be caused by inhomogeneities in the heterostructure. The temperature dependence of $B_{\mathrm{c}}$ is shown in Fig. 2, and $B_{\mathrm{c}}(T)$ increases with temperature in the $\mathrm{QH}$-conducting regime. This shows that $L_{\varphi}(T)$ decreases with temperature, thus implying the

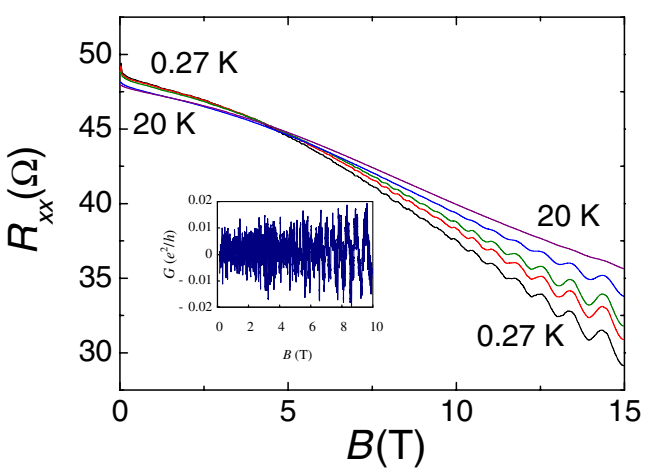

Fig. 1. (Color online) The longitudinal MRs of sample B at 0.27, 1.2, 3.1, 10.0 , and $20 \mathrm{~K}$ are shown in series. When temperature is increased, resistance increases in the region $B>5 \mathrm{~T}$ and decreases in the region $B<4 \mathrm{~T}$. The inset shows MR fluctuations at $0.27 \mathrm{~K}$.

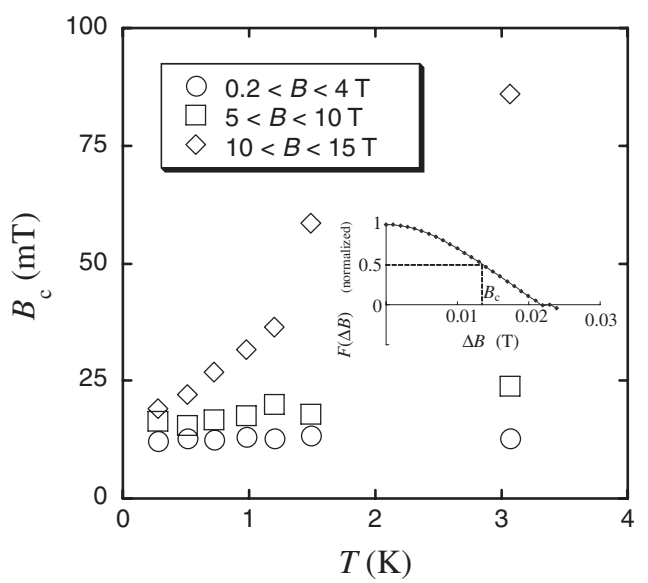

Fig. 2. Correlation fields $B_{\mathrm{c}}$ of sample B are plotted as a function of $T$. The three series for $0.2<B<4 \mathrm{~T}, 5<B<10 \mathrm{~T}$, and $10<B<15 \mathrm{~T}$ are marked by $\bigcirc, \square$, and $\diamond$, respectively. The inset shows an example of autocorrelation functions and correlation fields.

origin of quantum phase coherence in this phenomenon. However, the increase in $B_{\mathrm{c}}$ is not clear in the insulating regime, which may be caused by potential fluctuation by defects. To determine the effective temperature of carriers in hot-carrier phenomena, we also measured the current dependence of MR at the base temperature, and thus we obtained $B_{\mathrm{c}}(I)$, which is shown in Fig. 3. $B_{\mathrm{c}}(I)$ shows the tendency of increase in the $\mathrm{QH}$-conducting regime, but shows no clear increase in the insulating regime. We employed $B_{\mathrm{c}}$ as a self-thermometer, and thus obtained the effective carrier temperature in the $\mathrm{QH}$-conducting regime, which is shown in Fig. 4. The current scaling of carrier temperature shows the power law $T_{\mathrm{e}} \sim I^{0.5}$, which is similar to the result of previous theoretical and experimental studies of GaAs/AlGaAs and InGaAs/InP 2DESs. ${ }^{16,17,45)}$

\section{Conclusions}

In conclusion, we have presented experimental results of the electrical measurements of $\mathrm{GaN} / \mathrm{Al}_{0.15} \mathrm{Ga}_{0.85} \mathrm{~N}$ heterostructures grown on $\mathrm{Si}$. The $\mathrm{SiN}_{x}$ treatment enhanced the quality of the 2DES, where a clear SdH oscillation can be observed. We also employed autocorrelation analysis of conductance fluctuation, and thus obtained the correlation field $B_{\mathrm{c}}$ as function of $T_{\mathrm{e}}$ and $I$. In the $\mathrm{QH}$-conducting regime, $B_{\mathrm{c}}$ increases as $T_{\mathrm{e}}$ or $I$ increases. We also used $B_{\mathrm{c}}$ as a self-thermometer in the $\mathrm{QH}$-conducting regime, and the 


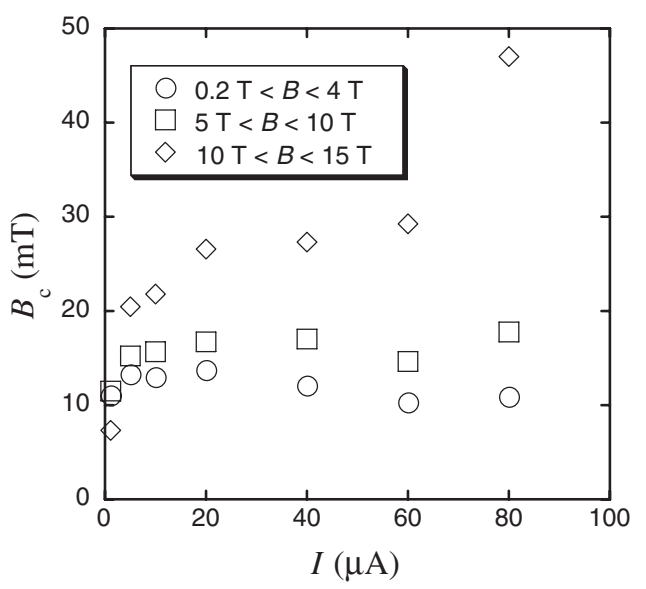

Fig. 3. Correlation fields $B_{\mathrm{c}}$ of sample B at base temperature are plotted as the functions of $I$. The three series for $0.2<B<4 \mathrm{~T}, 5<B<10 \mathrm{~T}$, and $10<B<15 \mathrm{~T}$ are marked by $\bigcirc, \square$, and $\diamond$, respectively.

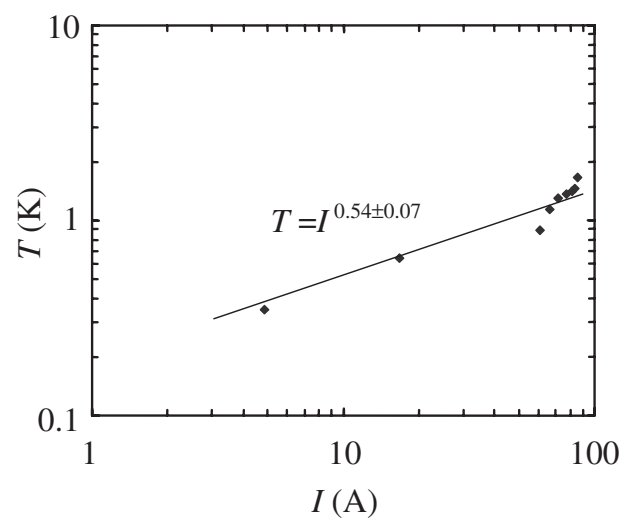

Fig. 4. Effective carrier temperature of sample B is plotted as a function of $I$, which is obtained from the results in Figs. 2 and 3. The straight line shows the power law $T_{\mathrm{e}} \sim I^{0.54}$.

current scaling of carrier temperature $T_{\mathrm{e}} \sim I^{0.5}$ is obtained, which agrees with the results of theoretical and experimental studies of GaAs/AlGaAs and InGaAs/InP 2DESs. ${ }^{16,17,45)}$

\section{Acknowledgement}

This work was funded by the National Science Council, Taiwan.

1) S. Nakamura, T. Mukai, and M. Senoh: J. Appl. Phys. 76 (1994) 8189.

2) S. Nakamura, M. Senoh, N. Iwasa, S. Nagahama, T. Yamada, and T. Mukai: Jpn. J. Appl. Phys. 34 (1995) L1332.

3) Y.-F. Wu, B. P. Keller, S. Keller, D. Kapolnek, P. Kozodoy, S. P. DenBaars, and U. K. Mishra: Appl. Phys. Lett. 69 (1996) 1438.

4) M. A. Khan, Q. Chen, M. S. Shur, B. T. Dermott, J. A. Higgins, J. Burm, W. J. Schaff, and L. F. Eastman: IEEE Electron Device Lett. 17 (1996) 584

5) M. P. Mack, A. Abare, M. Aizcorbe, P. Kozodoy, S. Keller, U. K. Mishra, L. Coldren, and S. DenBaars: MRS Internet J. Nitride Semicond. Res. 2 (1997) 41.

6) G. E. Bulman, K. Doverspike, S. T. Sheppard, T. W. Weeks, H. S. Kong, H. M. Dieringer, J. A. Edmond, J. D. Brown, J. T. Swindell, and J. F. Schetzina: Electron. Lett. 33 (1997) 1556.

7) C. R. Elsass, I. P. Smorchkova, B. Heying, E. Haus, P. Fini, K. Maranowski, J. P. Ibbetson, S. Keller, P. M. Petroff, S. P. DenBaars, U. K. Mishra, and J. S. Speck: Appl. Phys. Lett. 74 (1999) 3528.

8) I. P. Smorchkova, C. R. Elsass, J. P. Ibbetson, R. Ventury, B. Heying, P. Fini, E. Haus, S. P. DenBaars, J. S. Speck, and U. K. Mishra: J. Appl. Phys. 86 (1999) 4520.
9) L. K. Li, B. Turk, W. I. Wang, S. Syed, D. Simonian, and H. L. Stormer: Appl. Phys. Lett. 76 (2000) 742.

10) M. J. Manfra, L. N. Pfeiffer, K. W. West, H. L. Stormer, K. W. Baldwin, J. W. P. Hsu, D. V. Lang, and R. J. Molnar: Appl. Phys. Lett. 77 (2000) 2888.

11) T. W. Weeks, Jr., M. D. Bremser, K. S. Ailey, E. Carlson, W. G. Perry, and R. F. Davis: Appl. Phys. Lett. 67 (1995) 401.

12) A. Krost and A. Dadgar: Mater. Sci. Eng. B 93 (2002) 77.

13) K.-T. Wu, P. H. Chang, S. T. Lien, N. C. Chen, C.-A. Chang, C. F. Shih, W. C. Lien, Y. H. Wu, S.-C. Chen, Y. H. Chang, and C.-T Liang: Physica E 32 (2006) 566.

14) C.-A. Chang, S.-T. Lien, C.-H. Liu, C.-F. Shih, N. C. Chen, P.-H. Chang, H.-C. Peng, T.-Y. Tang, W.-C. Lien, Y.-H. Wu, K.-T. Wu, J.-W. Chen, C.-T. Liang, Y. F. Chen, T.-U. Lu, and T.-Y. Lin: Jpn. J. Appl. Phys. 45 (2006) 2516.

15) C.-T. Liang, K. Y. Chen, N. C. Chen, P. H. Chang, and C.-A. Chang: Appl. Phys. Lett. 89 (2006) 132107.

16) H. Scherer, L. Schweitzer, F. J. Ahlers, L. Bliek, R. Lösch, and W. Schlapp: Semicond. Sci. Technol. 10 (1995) 959.

17) H. P. Wei, L. W. Engel, and D. C. Tsui: Phys. Rev. B 50 (1994) 14609.

18) P. A. Lee, A. D. Stone, and H. Fukuyama: Phys. Rev. B 35 (1987) 1039

19) G. Timp, A. M. Chang, P. Mankiewich, R. Behringer, J. E. Cunningham, T. Y. Chang, and R. E. Howard: Phys. Rev. Lett. 59 (1987) 732.

20) A. K. Geim, P. C. Main, P. H. Beton, L. Eaves, S. P. Beaumont, and C. D. W. Wilkinson: Phys. Rev. Lett. 69 (1992) 1248.

21) F. Hohls, U. Zeitler, and R. J. Haug: Phys. Rev. B 66 (2002) 073304.

22) J. A. Simmons, S. W. Hwang, D. C. Tsui, H. P. Wei, L. W. Engel, and M. Shayegan: Phys. Rev. B 44 (1991) 12933.

23) P. C. Main, A. K. Geim, H. A. Carmona, C. V. Brown, T. J. Foster, R. Taboryski, and P. E. Lindelof: Phys. Rev. B 50 (1994) 4450.

24) A. A. Bykov, Z. D. Kvon, E. B. Ol'shanetskii, L. V. Litvin, and S. P. Moshchenko: Phys. Rev. B 54 (1996) 4464.

25) D. H. Cobden and E. Kogan: Phys. Rev. B 54 (1996) R17316.

26) D. H. Cobden, C. H. W. Barnes, and C. J. B. Ford: Phys. Rev. Lett. 82 (1999) 4695

27) T. Machida, S. Ishizuka, S. Komiyama, K. Muraki, and Y. Hirayama: Phys. Rev. B 63 (2001) 045318.

28) K. Ploog: J. Cryst. Growth 81 (1987) 304.

29) K. K. Choi, D. C. Tsui, and K. Alavi: Phys. Rev. B 36 (1987) 7751.

30) P. Mohanty, E. M. Q. Jariwala, and R. A. Webb: Phys. Rev. Lett. 78 (1997) 3366

31) P. Mohanty and R. A. Webb: Phys. Rev. Lett. 91 (2003) 066604.

32) R. G. van Veen, A. H. Verbruggen, E. van der Drift, F. Schäffler, and S. Radelaar: Semicond. Sci. Technol. 14 (1999) 508.

33) C. R. da Cunha, N. Aoki, T. Morimoto, Y. Ochiai, R. Akis, and D. K. Ferry: Appl. Phys. Lett. 89 (2006) 242109.

34) N. Aoki, D. Oonishi, Y. Iwase, Y. Ochiai, K. Ishibashi, Y. Aoyagi, and J. P. Bird: Appl. Phys. Lett. 80 (2002) 2970

35) T. Risaki, T. Ida, K. Ishibashi, and T. Sugano: Microelectron. Eng. 60 (2002) 347

36) J. P. Bird, A. Shailos, M. Elhassan, C. Prasad, K. M. Indlekofer, L. Shifren, R. Akis, D. K. Ferry, L.-H. Lin, N. Aoki, Y. Ochiai, K. Ishibashi, and Y. Aoyagi: Microelectron. Eng. 63 (2002) 277.

37) Y. Ochiai, L.-H. Lin, N. Aoki, J. P. Bird, A. Budiyono, K. Ishibashi, and K. Nakamura: Physica E 18 (2003) 147.

38) N. Aoki, L.-H. Lin, T. Morimoto, T. Sasaki, J.-F. Song, K. Ishibashi, J. P. Bird, A. Budiyono, K. Nakamura, T. Harayama, and Y. Ochiai: Physica E 22 (2004) 361.

39) M. W. Keller, A. Mittal, J. W. Sleight, R. G. Wheeler, D. E. Prober, R. N. Sacks, and H. Shtrikmann: Phys. Rev. B 53 (1996) R1693.

40) R. A. Jalabert, H. U. Baranger, and A. Douglas Stone: Phys. Rev. Lett. 65 (1990) 2442.

41) C. M. Marcus, A. J. Rimberg, R. M. Westervelt, P. F. Hopkins, and A. C. Gossard: Phys. Rev. Lett. 69 (1992) 506.

42) G. Benenti, G. Casati, I. Guarneri, and M. Terraneo: Phys. Rev. Lett. 87 (2001) 014101

43) M. Ferrier, L. Angers, A. C. H. Rowe, S. Guéron, H. Bouchiat, C Texier, G. Montambaux, and D. Mailly: Phys. Rev. Lett. 93 (2004) 246804.

44) D. G. Polyakov and B. I. Shklovskii: Phys. Rev. Lett. 70 (1993) 3796.

45) P. Z. Chen, L.-H. Lin, C.-T. Liang, J.-Y. Lin, J.-H. Chen, M. Y. Simmons, and D. A. Ritchie: J. Korean Phys. Soc. 50 (2007) 1662. 